\title{
Lapurdum
}

Euskal ikerketen aldizkaria | Revue d'études basques |

Revista de estudios vascos | Basque studies review

6 | 2001

Numéro VI

\section{Pour une critique contextuelle des « petites littératures » Bernardo Atxaga : l'invention de l'écrivain basque}

Ur Apalategi

\section{CpenEdition}

1 Journals

\section{Édition électronique}

URL : http://journals.openedition.org/lapurdum/1185

DOI : 10.4000/lapurdum.1185

ISSN : 1965-0655

Éditeur

IKER

Édition imprimée

Date de publication : 1 octobre 2001

Pagination : 7-18

ISBN : 2-84127-156-0

ISSN : $1273-3830$

Référence électronique

Ur Apalategi, «Pour une critique contextuelle des « petites littératures » Bernardo Atxaga : l'invention de l'écrivain basque », Lapurdum [En ligne], 6 | 2001, mis en ligne le 01 juin 2009, consulté le 03 février 2020. URL : http://journals.openedition.org/lapurdum/1185; DOI : 10.4000/lapurdum.1185 


\section{Ur Apalategi}

Maître de Conférences (Etudes basques)

à l'Université de Pau et des Pays de l'Adour

\section{Pour une critique contextuelle des "petites littératures"}

\section{Bernardo Atxaga : l'invention de l'écrivain basque}

S'arrachant peu à peu à "l'emprise originelle des instances politiques et nationales qu'elle a contribué à instituer et à légitimer"', la littérature, considérée comme champ sociologique transnational, tente continuellement de faire oublier cette paternité encombrante (la nation est une création littéraire) qui la leste et la diminue autant, sinon davantage, qu'elle la glorifie. Pourtant, le processus d'autonomisation de la littérature se réalise paradoxalement nation par nation. "La conquête de la liberté de l'ensemble de l'espace littéraire mondial s'accomplit donc à travers l'autonomisation de chaque champ littéraire national : les luttes et leurs enjeux se délivrent des impositions politiques pour ne plus obéir qu'à la seule loi spécifique de la littérature"2. Autrement dit, la dénationalisation (l'universalisation) de la littérature a partie liée avec la constitution d'un espace national. Ceci explique sans doute l'horreur de l'extra-littéraire, a fortiori dans les littératures des nations fragiles ou sans Etat, encore ombilicalement rattachées à leur raison d'être première, la raison nationale.

La littérature basque fait partie de ces éternels aspirants à l'autonomie vis-à-vis du champ politique national. De ce fait, elle partage avec les autres aspirants une même angoisse existentielle. Sans doute pourrait-on thématiser cette «angoisse » de la façon suivante : la pratique de la littérature littéraire repose matériellement sur la pérennité de la langue nationale; or, la survie de la langue dépend de celle de la nation (et, plus concrètement, de la mise en place d'institutions étatiques telles que le système éducatif, producteur du lectorat); par conséquent, les écrivains aspirant à l'autonomie de leur art doi-

\footnotetext{
' Pascale Casanova, La République mondiale des lettres, Paris, Seuil, 1999, p.60.

${ }^{2}$ Ibid., p.61.
} 
vent d'abord se battre pour la survie de la nation, ce qui est, on s'en doute, en totale contradiction avec leur souhait initial d'autonomisation littéraire. C'est dire la difficulté d'une critique littéraire contextuelle appliquée à ces petites traditions littéraires extrêmement soucieuses d'assurer leur faible autonomie acquise à dures peines et sans cesse remise en question. En effet, toute entreprise critique de ce type risque à tout moment de dévoiler la vérité à peine cachée de l'hétéronomie plus ou moins grande dans laquelle vit l'institution littéraire. Les représentations de la littérature que les écrivains des nations du « tiers-monde littéraire » essaient de propager dans la société - et par l'entremise desquelles ils font un travail d'autosuggestion destiné avant-tout à eux-mêmes - résistent mal au regard contextuel. Mais c'est précisément la fragilité de telles institutions sociales, l'issue incertaine du combat qu'elles livrent qui en rend l'étude précieuse.

\section{Un acteur de la vie littéraire basque}

Bernardo Atxaga (de son vrai nom, Joseba Irazu Garmendia) est né en 1951 à Asteasu, petit village de moyenne montagne de la province de Guipuzcoa, au Pays Basque d'Espagne. A l'époque où il commence à publier ses premiers écrits en euskara (1972), le régime franquiste en place interdit toute manifestation culturelle en langue basque. La prudence impose à beaucoup d'écrivains espagnols (non seulement basques) le choix d'un pseudonyme pour pouvoir publier leurs œuvres. Au milieu de l'effervescence idéologique qui s'étend à tous les secteurs de l'opposition au régime, la "littérature sociale" ou "engagée" vit ses heures de gloire. Mais, déjà, de nouvelles générations d'auteurs ayant eu accès à la "bibliothèque universelle" et s'en étant nourris viennent frapper à la porte. Des courants et des groupes littéraires se forment qui entendent délivrer la littérature de son asservissement utilitariste. Parmi ces derniers on trouve, au Pays Basque, le groupe Pott dont Atxaga est précisément l'un des fondateurs. Atxaga fait volontairement dérailler le train de la littérature basque de la voie toute tracée du réalisme ou de l'allégorie politique en y introduisant le surréalisme, l'oulipisme et autres courants avant-gardistes. Puis, délaissant la stratégie élitiste mise en œuvre jusque-là (à coup de manifestes et d'œuvres hermétiques), Atxaga se tourne au cours des années 80 vers la littérature pour enfants (seul véritable marché littéraire bascophone grâce à l'introduction de la langue basque dans le système éducatif de la Communauté Autonome Basque), genre moins prestigieux mais lui permettant de se professionnaliser. Atxaga conçoit la professionnalisation comme le meilleur moyen d'affirmer l'autonomie de I'activité littéraire face 
à l'omniprésence du discours politique (toutes tendances confondues) qui se constitue autour de ce que l'on nomme déjà « le problème basque ». Le destin littéraire d'Atxaga se voit bouleversé par l'attribution du Prix National de Littérature d'Espagne (1989) à son œuvre Obabakoak (rédigée en langue basque et traduite pour l'occasion) et la consécration internationale qui s'ensuit (l'œuvre sera traduite dans plus de vingt langues). La littérature basque tient en lui son premier auteur universel et en voit sa légitimité renforcée au sein de l'espace social basque. Les années qui suivent ce changement de statut spectaculaire verront la reconfiguration totale de la problématique littéraire de l'auteur, reconfiguration s'accompagnant d'une réorientation stylistico-thématique parallèle.

On se propose ici de travailler à une interprétation de la problématique existentielle du personnage-type du corpus atxaguien (postérieur à cette date charnière) qui tienne compte de la modification contextuelle de la situation d'énonciation dans laquelle de trouve l'auteur. A cet effet, la production littéraire de l'auteur sera considérée comme l'objet d'un discours polyphonique (celui de l'auteur, celui des divers récepteurs - journalistes, critiques -, le nôtre) dont l'enjeu est bien d'en fixer le sens.

\section{Problématique de l'auteur dénationalisé}

Le personnage atxaguien récurrent du corpus des années 90, développant une philosophie personnelle imprégnée d'individualisme, a préféré la solitude (idéologique et relationnelle) à l'embrigadement ${ }^{3}$. Il se situe dans un entre-deux spatial (d'un côté Barcelone, ou d'autres endroits de la géographie espagnole pouvant symboliser le marché littéraire espagnol et/ou international, et de l'autre la littérature basque, tour à tour et métonymiquement symbolisée par Bilbao, Irurzun, Asteasu ou le village imaginaire d'Obaba). Les rapports qui unissent le personnage à chacune des deux parcelles de cet espace sont d'ordres divers mais ont pour trait commun leur caractère ambigu, ambivalent et insuffisant. En effet, le personnage finit toujours par refuser sa fidélité à quelque parcelle de l'espace que ce soit. L'affirmation de son individualité souveraine mais douloureuse l'emporte sur une fidélité spatiale liée

\footnotetext{
${ }^{3}$ Le roman paradigmatique de la production atxaguienne des années 90 porte un titre éclairant L'Homme seul (Paris, Christian Bourgois, 1995) - et narre le devenir incertain d'un ancien militant de I'ETA, reconverti dans l'hôtellerie à Barcelone et qui met sa situation en danger Iorsqu'il décide, sans avertir ses associés - également activistes à la retraite -, d'héberger des militants en fuite dans la cave de la boulangerie de l'hôtel.
} 
à un sens collectif et à une protection communautaire. Souffrance et stratégie s'associent dans la tentative permanente du personnage d'échapper à l'obligation de choisir entre les deux camps sans en pâtir les graves conséquences (l'ostracisme et la mort) qui lui sont promises.

Il s'agit maintenant de mettre en relation cette problématique avec la situation positionnelle dans laquelle Atxaga évolue. La spécificité absolue de la position de l'écrivain résulte de sa double inscription simultanée dans des champs sociologiques concurrents. Il aurait pu laisser le soin à un traducteur professionnel de traduire son œuvre en espagnol, ce qui aurait fait de lui un auteur s'inscrivant exclusivement dans le champ littéraire basque et bénéficiant d'une traduction à titre d'auteur "étranger". Au lieu de cela, il a préféré prendre en charge la traduction de sa propre œuvre, ce qui fait de lui un auteur de plein droit du champ littéraire espagnol ${ }^{+}$. La double inscription sociologique d'Atxaga n'est pas sans conséquences sur le travail d'interprétation de ses œuvres. On peut considérer qu'à partir du roman L'Homme seul l'auteur semble avoir pris définitivement conscience qu'il s'adresse simultanément à deux publics aux caractéristiques opposées. L'assurance qu'il a acquise de voir dorénavant chacune de ses créations traduites et publiée par les maisons d'édition espagnoles modifie radicalement sa proprioception positionnelle antérieure de simple auteur basque. Le découpage symbolique dichotomique de l'espace (Barcelone/Pays Basque) et la situation topologique intermédiaire et ambiguë du personnage central, caractéristiques de la problématique biculturelle qui est celle de l'auteur s'inscrivant dans deux champs littéraires concurrents, contraste nettement avec l'unicité de l'espace et la situation dominante du personnage central dans une œuvre telle que Mémoires d'une vache, écrite à une époque (1990) où l'auteur se perçoit encore comme appartenant exclusivement au domaine littéraire basque. La suppression de l'adjectif "basque" (le titre de la version originelle basque de l'œuvre est "Mémoires d'une vache basque") lors de la traduction tend à prouver que le livre fut conçu pour un destinataire basque et que l'écrivain a jugé préférable, devant la perspective de le publier en espagnol (puis en français et en d'autres langues ${ }^{5}$ ), d'éviter des lectures réductrices de l'œuvre et de favoriser le pro-

\footnotetext{
+ Tel n'est pas le cas, par exemple. d’A. Lertxundi - finaliste du Prix National de Littérature d'Espagne 1994 - qui explique qu'il préfère recourir à un traducteur afin de pouvoir consacrer entièrement son temps à la création (en langue basque).

5 Il est utile de souligner que les traductions dans les langues autres que l'espagnol (français, anglais, etc.) se font à partir de la version castillane des œuvres effectuée par l'auteur. faute de traducteurs qualifiés pouvant le faire à partir du texte originel en langue basque (et. sans doute également, en raison de l'inexistence d'un rayon « littérature basque » dans les librairies, ce qui pousse les éditeurs à préférer l'étiquette. plus commerciale. de « domaine hispanique »).
} 
cessus d'identification du lecteur espagnol, puis international, au personnage principal.

A partir de la prise de conscience par l'écrivain de sa double inscription sociologique, on peut considérer que les conditions de l'énonciation lit= téraire ont changé. La modification de la nature de l'un des trois termes formant le triangle énonciatif mis en lumière par la pragmatique (destinateur/œuvre/destinataire) provoque une altération sensible de l'essence même de la communication littéraire. Chacun des motifs d'une œuvre s'adresse consciemment à deux lectorats aux caractéristiques opposées. L'auteur sait que chacune des lignes qu'il rédige sera ensuite "traduite" par lui-même et parviendra à un second lectorat ${ }^{6}$ ayant des caractéristiques opposées à celles du premier et "recevant" le texte avec un "horizon d'attente" formé par un héritage intertextuel radicalement différent. Il est loisible de penser que chaque motif inséré par l'auteur dans la trame de son œuvre est chargé non pas d'une intentionnalité unique (comme dans le cas des œuvres écrites pour un seul lectorat homogène) mais d'une intentionnalité double ou multiple découlant de la prise en compte au moment créateur de la présence d'un destinataire double ou multiple. C'est ce que nous nommons la pluri-intentionnalité du motif littéraire. Cela entraîne une interprétation double et réversible qui peut s'appliquer au passage de la nouvelle Reflexiones un tanto francesas dans lequel le narrateur-écrivain dénonce la conception nationalitaire de la littérature qui prédomine dans la plupart des pays :

[...] dans la plupart des pays du monde le Ministère de l'Éducation conçoit la littérature comme littérature "nationale", et oblige des millions d'écoliers à étudier une histoire de la littérature qui est toujours celle de l'Espagne et seulement celle de l'Espagne, ou celle de la France et seulement celle de la France, en réduisant au silence les cours où l'on enseignait l'Histoire de la Littérature Universelle. ${ }^{7}$

\footnotetext{
"Wll serait préférable d’employer l'expression d'"écriture répétée" ou le tẹme de réécriture (bien que son emploi soit ici équivoque) afin de bien rendre compte du fait que chacun des deux destinataires lira un texte qui lui est destiné, qui a été créé en fonction de sa présence, et non un texte s'adressant primitivement à un autre lectorat et qui par la suite a été traduit.

${ }^{7}$ B. Atxaga, "Reflexiones un tanto francesas", in Horas extra, Madrid, Alianza, 1997, p.85 (nous (raduisons),
} 
Si la remarque s'applique au monde littéraire espagnol ou au marché international - dans lesquels le produit Atxaga a été vendu sous l'étiquette « nouvelle Espagne, exotisme rural basque » - ne doutons pas qu'elle s'applique également (dans l'esprit et l'intention de l'auteur) au monde littéraire basque auquel l'auteur reproche le même travers. Les acteurs de la vie littéraire basque commencent à comprendre, au moment de la publication du texte, que celui par qui le reclassement est arrivé mène en quelque sorte une double vie et risque par cette attitude de réduire à néant le gain symbolique collectif que sa consécration individuelle a supposé pour eux-mêmes. Le ressentiment et la jalousie s'installent au cœur de la relation qui unit Atxaga à son domaine littéraire originel. L'auteur déclare à plusieurs reprises, et dans divers médias, qu'il refuse de devenir un "écrivain national" " pas disposé à sacrifier son parcours individuel au profit d'une littérature basque ayant grandement besoin du capital symbolique que représente désormais l'écrivain internationalement consacré qu'il est devenu. Cette réticence d'Atxaga n'est pas sans rappeler celle du militant à la retraite Carlos - «homme seul »-qui prend le soin de spécifier, au cours d'un entretien avec Jone - le membre féminin du couple d'activistes qu'il héberge dans la cave de sa boulangerie -, que sa décision est ponctuelle et qu'elle ne signifie en aucune façon qu'il continue à se sentir membre de l'organisation clandestine:

"Actuellement, je vous cache, d'accord, parce que, quand on me l'a demandé, il m'a semblé que je devais le faire. Mais ce n'est pas une décision qui vaut pour l'éternité. Il se peut que la prochaine fois je refuse. Il faut que vous vous mettiez ça dans la tête. Moi, je ne fais pas partie de l'organisation" 9

Atxaga refuse de se sentir lié de manière exclusive au domaine littéraire basque en vertu d'un supposé contrat moral tacite et on lui en tient rigueur. Il devine que la relation que l'organisation - la littérature basque entend soudainement approfondir avec lui est intéressée. Le monde littéraire basque compte profiter de la position acquise par lui sur le marché espagnol afin d'assurer et de consolider son propre reclassement à l'intérieur de l'espa-

\footnotetext{
8 "Yo no soy el escritor nacional de Euskadi", La Revista, 21-7-1996. Madrid. p. 16-23.

${ }^{9}$ B. Atxaga, L'Homme seul. op. cil., p.44-45 éd. française (nous soulignons).
} 
ce social basque. Carlos impute à l'activiste Jone un raisonnement qui rend bien compte de la nature intéressée de la sollicitude dont il fait l'objet de la part de l'organisation :

"Il s'en faut de très peu pour que cet homme ne revienne dans l'organisation, et c'est pourquoi il a accepté de nous cacher, Jon et moi. Et ce serait pour nous une aubaine s'il arrivait à faire le pas, parce qu'il dispose de la meilleure cachette du monde, un hôtel situé à quelques kilomètres de Barcelone dans un endroit pratiquement désert. Un succès magnifique qui résoudrait beaucoup de problèmes d'infrastructure". 10

\section{Entre engagement et compromission}

L'auteur s'interroge, tout au long de la décennie, sur la nature du lien qui l'unit malgré tout à la littérature basque. Carlos perçoit la mission d'hébergement qu'il doit mener à bien comme une épreuve libératrice après laquelle sa conscience ne lui posera plus de problèmes et il pourra s'intégrer pleinement à une vie barcelonaise nouvelle (il projette d'apprendre le catalan et de se défaire de sa personnalité actuelle, conditionnée par son appartenance passée à l'organisation). Autrement dit, Atxaga nourrit le secret espoir que s'il arrive à régler une fois pour toutes le problème de sa prétendue dette envers la littérature basque, il pourra s'intégrer pleinement à la vie littéraire espagnole ou internationale (symbolisée par Barcelone) ${ }^{11}$.

L'intrusion inespérée d'un tiers élément dans le rapport sadomasochiste qui unissait l'auteur autonomisant à une petite tradition littéraire dominée par une conception engagée de la littérature provoque inévitablement la jalousie collective à l'égard d'un auteur désormais accusé par certains de ses confrères de s'être laissé séduire par l'"autre". Dans le quotidien Egunkaria, la journaliste Ines Intxausti commentant une intervention récente de l'auteur à la télévision basque destinée aux hispanophones ${ }^{12}$ souligne l'aisance de l'auteur cons-

\footnotetext{
${ }^{10}$ Ibid., p.43.

" Barcelone présente l'avantage - face à sa concurrente Madrid - d'être en même temps la capitale littéraire espagnole (en raison de sa concentration éditoriale) ainsi qu'une capitale littéraire dénationalisée (en raison de son passé artistique cosmopolite). Elle est, pourtant, le lieu pouvant le mieux symboliser la place d'Atxaga sur l'échiquier littéraire mondial.

${ }^{12}$ La Télévision basque (Euskal Telebista, ETB) possède deux chânes généralistes : l'une diffuse en basque, l'autre en espagnol.
} 
cient de s'adresser à l'ensemble de la population du Pays Basque (bascophones et non-basccophones confondus), et compare cette aisance à la tension qui se lit sur son visage chaque fois qu'il s'adresse à la seule communauté bascophone:

[...] il ne semblait pas qu'il fût aussi mal à l'aise que le jour où il répondit aux questions de J. Garzia et de $\mathrm{H}$. Etxeberria [sur le canal bascophone]. Et le problème ne résidait nullement [...] dans l'identité des ces deux présentateurs, mais dans la présence de cette audience basque qu'une relation d'amour - désir - haine unit à B. Atxaga. [...] Enfin, il allait être écouté [sur le canal hispanophone] par ceux auxquels il désirait s'adresser. Et non par les jaloux de toujours qui ne pouvaient s'empêcher d'éprouver du ressentiment $[\ldots]{ }^{13}$

Des propos plus récents de l'écrivain Pako Aristi traduisent le désenchantement d'une littérature basque qui se voit progressivement délaissée par l'artisan majeur de son reclassement sociologique. L'article commente la parution du recueil Horas extras (Madrid, Alianza, 1997) qui n'a été écrit que dans sa version castillane. Aristi s'y déclare surpris par la manière dont Atxaga décrit le Pays Basque, manière qu'il qualifie de froide et plus propre de quelqu'un qui est de passage que de quelqu'un qui y habite et qui sent les choses qui s'y déroulent. Constatant que le livre a été conçu pour le lectorat espagnol dans son ensemble, il déplore le fait qu'Atxaga ne fasse pas grand cas de la présence au Pays Basque (où le livre sera lu par les deux communautés qui coexistent difficilement) d'une communauté bascophone qui est porteuse d'une vision différente de la réalité et qui souffre habituellement d'un lourd silence médiatique dans les médias hispanophones. Selon Aristi, les hispanophones du Pays Basque ne montrent aucun intérêt pour ce qui est produit en langue basque, mis à part pour ce qui touche à B. Atxaga. La déception n'en est que plus grande :

Je pense qu'il est actuellement le seul pont interculturel ai Pays Basque, et c'est pourquoi son conformisme m'est douloureux : au lieu d'aider les hispanophones à découvrir une autre réalité, il les confirme dans leurs opinions antérieures [...]. Je ne sais si cela en est la cause,

13 I. Intxausti, "Bernardo Atxaga Forumean", Egunkaria 9 septembre 1997. Andoain, p.33. 
mais il me semble que les écrits, idées ou propositions de B. Atxaga ne sont plus tellement commentées au sein de la communauté bascophone, ces derniers temps ${ }^{1+}$.

Il n'est pas difficile d'établir une chronologie sommaire mais éclairante de ce processus d'éloignement : Obabakoak (1989) fut rédigée en langue basque, puis expressément traduite par l'auteur, assisté dans cette tâche par d'autres écrivains-traducteurs, à seule fin de la présenter au Prix National de Littérature d'Espagne; Mémoires d'une vache (1991), fut écrite d'abord pour un destinataire bascophone, ce que les plaintes postérieures de l'auteur concernant l'accueil frileux réservé à l'œuvre par le lectorat basque prouvent sans peine; en 1993, Atxaga publie le roman L'Homme seul dont il écrit simultanément les deux versions; en 1996, pour l'œuvre Un Homme nommé Sara, fait jusque-là inédit, la publication de la version en castillan précède la publication de la version en langue basque; enfin, en 1997, Atxaga publie le recueil de nouvelles Horas extras qui est manifestement destiné, comme Aristi le souligne (voir supra), au lectorat hispanophone du Pays Basque ou du reste de l'Espagne. En réalité, Atxaga est à la recherche d'une motivation réelle pour continuer à écrire en langue basque :

Ce serait formidable de trouver un filon, c'est-à-dire de trouver une raison solidement fondée qui justifierait que l'on continue à écrire en basque. ${ }^{15}$

Comment apprécier, après avoir connu la douceur du « statut d'écrivain hispanique traduit dans le monde entier », l'étroitesse et l'inconfort d'une littérature basque incomplètement structurée et dont l'autonomie sera toujours fragile tant que l'assise sociolinguistique de la langue basque ne sera pas stabilisée?

Toutefois, il faut se garder de conclure que l'écrivain trouve dans la position qu'il lui échoit d'occuper au sein du paysage littéraire espagnol ce à quoi il aspirait réellement. Dans la nouvelle Reflexiones un tanto francesas,

\footnotetext{
14 P. Aristi, "Ordu estrak", Egunkaria 5 septembre 1997, Andoain, p.2 (nous traduisons).

15 Ibid., p.30 (nous traduisons toujours).
} 
l'auteur poursuit son auto-analyse par l'entremise d'une fable ${ }^{16}$. À la question "quel est l'animal le plus cruel?", les insectes (animaux les plus faibles, symbolisant l'écrivain) répondent sans hésiter qu'il s'agit des moutons (dont les insectes sont la pâture) et à la question "quel est l'animal qui se caractérise par sa bonté?" ces mêmes insectes répondent sans hésiter que cet animal-là est le tigre (qui venge les insectes en pourchassant le mouton). Cet apologue sert à exprimer le paradoxe de la position atxaguienne dans l'espace social : du point de vue purement subjectif et relatif qui est le sien, l'auteur ne peut s'empêcher de penser que le marché littéraire espagnol (le tigre) lui a rendu un grand service en court-circuitant l'emprise que la littérature basque (le mouton) soumise à l'influence du champ politique avait sur lui (insecte fatigué par tant d'années passées à défendre l'autonomie de la littérature). Nonobstant, Atxaga ne se fait guère d'illusions ni sur la manière dont il s'est laissé séduire par la littérature espagnole ni même sur le rôle que celleci lui réserve. Les réflexions que le personnage d'écrivain de la nouvelle développe montrent la façon dont l'auteur s'identifie avec clairvoyance aux écrivains du tiers-monde auxquels on assigne un rôle précis dans le système littéraire international :

[...] "exotique". C'est ce que, littérairement parlant, le Premier Monde exige du Second ou du Tiers-monde : qu'il soit exotique. ${ }^{17}$

N'oublions tout de même pas qu'Atxaga a été récompensé pour une cuuvre, Obabakoak, qui se présentait, entre autres - et bien que cet aspect de l'œuvre soit d'un intérêt secondaire -, comme une exploration du passé ethnographique du Pays Basque, comme une approche littéraire de l'inconscient collectif de la communauté bascophone. L'auteur, lui, ne semble pas avoir oublié que c'est Obabakoak (œuvre néo-ruraliste) qui a reçu le Prix National et non L'Homme seul. Ses textes-essais de 1997 (recueillis dans Horas extras) démontrent, si besoin était, qu'il en a tiré des leçons. L'euphorie consécutive à l'attribution du Prix et à l'adaptation à sa nouvelle vie d'écrivain internationalement reconnu étant passée, il entreprend une analyse sereine et désenchantée du rôle qui lui échoit. Esquissant une théorie de la structure du marché littéraire mondialisé, Atxaga brosse le portrait sans

\footnotetext{
16 B. Atxaga. "Reflexiones un tanto francesas", in Horras extras, op. cit. p.77-78.

17 B. Atxaga, "Reflexiones un tanto francesas", in Horas extras. op. cit., p.93 (nous traduisons).
} 
concessions de l'écrivain-type d'un pays littérairement (et souvent littéralement) pauvre. Celui-ci se doit d'être le fier représentant des spécificités locales dont il ferait la matière première de ses œuvres. Mais les exigences du premier monde littéraire ne s'arrêtent pas là, puisque l'auteur "périphérique" se voit interdire l'accès à certains genres, chasses gardées des écrivains du "centre" :

Il serait encore plus surprenant que le marché éditorial européen, ou bien le public lui-même, acceptent qu'un de ces écrivains fasse les mêmes choix que les écrivains de la métropole. "Nous avons déjà des romans intellectuels, des romans sophistiqués, des romans avant-gardistes", dirait la métropole, "ce dont nous avons besoin c'est d'une littérature de la mémoire, de nouvelles de la marge. Un peu d'exotisme, s'il vous plaît, ou à défaut, de la littérature revendicative, l'histoire de la grande souffrance qui vous est infligée par votre dictateur". 18

Admirable tentative d'objectivation que celle à iaquelle se livre Atxaga. L'auteur, dont le Cycle d'Obaba va à l'encontre de l'idée même d'exotisme - Obabakoak doit être lue comme une violente diatribe contre l'idéologie culturaliste et non comme une exaltation nostalgique de quelque essence identitaire collective, inconsciente ou archaïque (selon les goûts) comprend, avec dépit, que son œuvre n'a pas nécessairement été récompensée pour les bonnes raisons et que "la reconnaissance des capitales littéraires se fait au prix d'une extraordinaire annexion de l'œuvre excentrée aux intérêts centraux" 19 . Il en résulte qu'Atxaga ne semble destiné à occuper, au sein de la vie littéraire espagnole, qu'une position dominée et périphérique de représentant patenté de la parcelle basque de l'identité plurielle de l'Espagne contemporaine. Lui qui s’est battu deux décennies durant, au sein de la littérature basque, contre une conception nationalitaire de la littérature en tant que vecteur de l'identité collective se retrouve de nouveau enfermé dans une catégorie nationale. D'un côté, le monde littéraire basque prétend faire de lui le héros et le héraut du reclassement collectif de l'institution, de l'autre le monde littéraire espagnol entend le confiner à ce rôle d'écrivain « national » qu'il abhorre :

\footnotetext{
${ }^{18}$ B. Atxaga, "Horas extras", in Horas extras, op. cit., p.43.

19 P. Casanova, La République mondiale des Lettres, op. cit., p.226.
} 
Longtemps l'on a soutenu que celui qui est basque est nationaliste, partant que I'écrivain basque est forcément nationaliste. Cet amalgame a été recherché, de façon intéressée, des deux côtés de la tranchée. Eh bien, je n'y souscris pas. 20

\section{Bibliographie}

APALATEGUI, U., La Naissance de l'écrivain basque. L'évolution de la problématique littéraire de Bernardo Atxaga, Paris, l'Harmattan, 2000, 299 p.

ARISTI. P.. "Ordu estrak", in quotidien Egunkaria 5 septembre 1997. Andoain (Guipuzcoa).

ATXAGA, B., Obabakoak, Paris, Christian Bourgois, 1990 (Donostia-San Sebastián, Erein. 1988, pour la version originelle en langue basque).

ATXAGA. B., Mémoires d'une vache. Paris. Gallimard, 1994 (pour la version originelle en langue basque, Behi euskaldun baten memoriak, Iruñea-Pamplona, Pamiela, 1991).

ATXAGA, B., L'Homme seul. Paris, Christian Bourgois. 1995, (pour la première édition en langue basque. Gizona bere bakardadean, Iruñea-Pamplona, Pamiela, 1993).

ATXAGA, B.. "Yo no soy el escritor nacional de Euskadi". La Revista. 21-7-1996, Madrid.

ATXAGA, B., Horas extra, Madrid, Alianza, 1997.

BOURDIEU, P., Les Règles de l'art, Paris, Seuil, 1992.

CASAnOVA, P., La République mondiale des lettres, Paris. Seuil, 1999.

INTXAUSTI, I., "Bernardo Atxaga Forumean". in quotidien Egunkaria 9 septembre 1997. Andoain (Guipuzcoa).

VIALA, A., Naissance de l'écrivain, sociologie de la littérature à l'âge classique, Paris, Éditions de Minuit, collection Le Sens commun, 1985.

20 In entrevue "Yo no soy el escritor nacional...". op. cit. p. 23 (nous soulignons) 